THERMAL MICROSENSORS

\section{Widespread Applications}

The Europhysics Industrial Workshop Thermal Microsensors: Their Bases, Principles and Applications held at the Hotel Panorama in Oberhof, Germany, on 25-28 April 1994 clearly promoted a stronger collaboration between fundamental research and industry in the field.

The number of meetings, publications, exhibitions and the amount of research funding indicates that there has been a growing interest in microsensors in recent years. This trend also applies to thermal microsensors (see insert) where a non-thermal signal is converted into an on-chip temperature gradient. The gradient can be detected by applying an appropriate physical effect, e.g., the thermoresistivity, to convert the temperature difference into an electric signal.

This physically simple functional principle results in rugged and low-priced microsensors suitable for many applications including infrared thermometry and pyrometry, object registration and intrusion alarms as well as for heat flux measurements and gas analysis.

The Europhysics Industrial Workshop offered an excellent opportunity to review the state-of-the-art in the field of thermal microsensors, where there are currently several different kind of sensors including infra-red radiation detectors (thermopiles, bolometers, pyroelectric sensors), microwave power sensors and AC-DC thermal converters, fluid flow and vacuum sensors, accelerometers, thermal conductivity gauges, micro-calorimeters, and psychrometers. The sensors are generally miniaturized using micro-mechanical and photolithographic technologies, and the design and layout of the microsensor chips is often optimized by carrying out thermal modelling to simulate the temperature distribution and heat flow. Since modelling of the complete sensor may be too expensive, it is often restricted to the most important functional parts such as the free-standing membranes, cantilever beams, etc. of the sensors.
While simulation using computers represents one way of improving the performance (e.g., response, sensitivity, time constant) of thermal microsensors, another way is the proper choice of materials for the various functional layers. For a high sensitivity, the most important component is the materia used in exploiting the Seebeck effect to detect the temperature gradient across the sensor's membrane. Materials ensuring an effective thermoelectric energy conversion at room temperature are semi-metals such as $\mathrm{Bi}, \mathrm{Sb}$ and their alloys and $\mathrm{V}-\mathrm{VI}$ compound semiconductors such as bismuth-antimony tellurides/selenides and lead telluride.

Complex metal oxide on silicon (CMOS) technology has seen almost 20 years of development, so suitably doped polycrystalline silicon as a CMOS-compatible material represents another key material for thermal microsensors. Manufacturing silicon-based fully CMOS-compatible thermal sensors is most advantageous if there exists a mass market, whereas sensors fabricated using non-CMOS-compatible technologies have to meet special customer requirements to fill market niches. While the symbiosis with CMOS circuits and CMOS technology is introducing important advantages in terns of cost and system performance, there are also others trends. In particular:

\title{
Thermal Microsensors
}

Thermal microsensors generally monitor either the power needed to maintain a temperature difference (thermal power sensor) or the response of the sensor's temperature to the parameter being measured (thermal resistance sensor). Many different configurations are possible since the effective diffusivities for the heat-flow mechanisms involved (infra-red radiation, phase change, conduction, convection) vary by 5 6 orders of magnitude. Generally speaking, however, the temperature difference for a power sensor is created on a thin membrane (to permit an accurate determination) integrated into an electronic chip (to allow small dimensions). The three main types of membranes are: closed (supported on all sides - see Fig. 1a); lightly suspended or floating, where the active part is supported by a few bridges; cantilever beam-like, where a thin beam protrudes from the supporting structure.

Micromachining technologies compatible with the basic processes used for fabricating integrated electronic circuits are generally used. This permits active electronic circuits such as heating resistors and thermopiles to be integrated into the membrane or cantilever (Fig. 1a,b). High sensitivity to measure, for instance, tracegas concentrations requires highly efficient thermoelectric materials arranged in complex, multi-element thermopile arrays on the membrane (Fig. 1c). Several membrane-based thermal microsensors can be incorporated on the same chip together with on-chip circuitry. (Fig. 1d), and power may be introduced using other techniques (e.g., by coupling to an RF field to measure various quantities). Finally, the thermal microsensor must be packaged (Fig. 1e) and mounted (Fig. 1f).

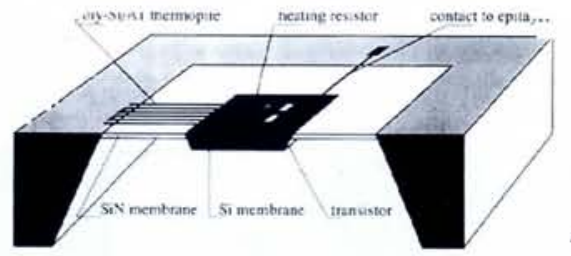

a)

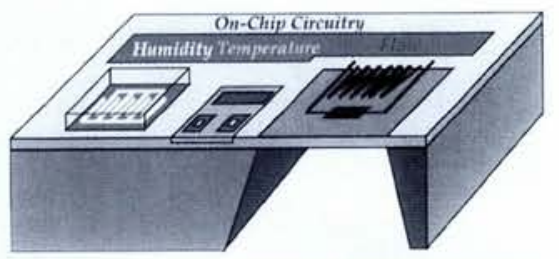

d)
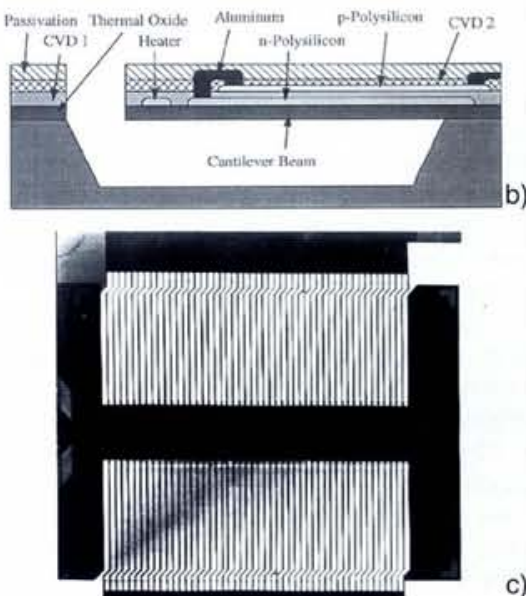

c)

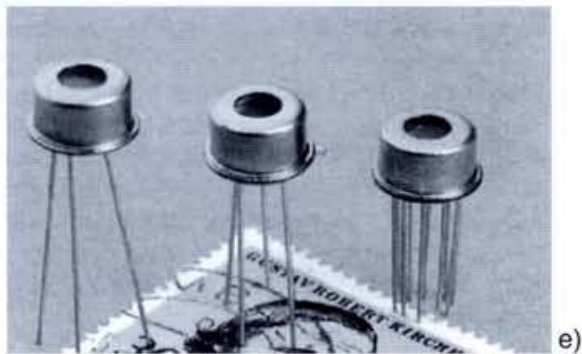

e)

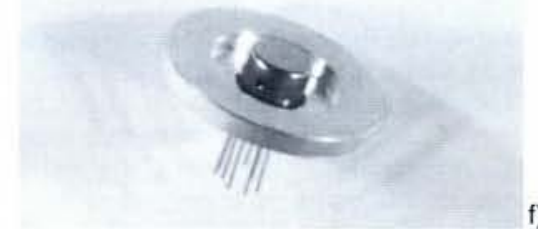

Fig. 1 - Thermal microsensors. a) A schematic illustration of an advanced thermal power sensor from Xensor Integration bv, Delft. The silicon membrane is itself supported by a SiN membrane to combine the high thermal insulation of SiN with the possibility of using bipolar Si-based IC devices as the sensing elements. An electrical signal fed into the resistor on the Si membrane and the resulting heat flow to the bulk chip across the membrane creates a temperature difference that is converted, via the Seebeck effect, into a DC signal by a thermopile.

b) A section through a thermopile cantilever fabricated in bidoped double-polysilicon using the CMOS process ( $H$. Baltes, ETH Zurich).

c) A scanning electron micrograph of the supported membrane of a micromachined highsensitivity, thermal convertor chip developed by the IPHT, Jena. The upper $2 \times 50$ legs of the thermopile are shown; the remainder, separated by an insulating layer, lie underneath.

d) A schematic illustration of a chip combining several sensors that is intended to control the humidity, temperature and flow velocity of air in buildings ( $H$. Baltes, ETH Zurich).

e) Packaged infra-red microsensors.

f) A flange-mounted vacuum sensor (Xensor Integration bv, Delft) based on a thermal microsensor which measures the thermal conductivity of the vacuum gas. 


\section{EIW-10: Thermal Microsensors}

The idea of organizing a Europhysics Industrial Workshop devoted to thermal microsensors was born at the Institute for Physical High Technology (IPHT), the former Physical-Technical Institute of the eas German Academy of Sciences, were a thermal sensors group has been active for many years. It was taken up by other groups working in the field, notably those at the Swiss Federal Institute of Technology (ETH) in Zurich and at Heimann Optoelectronics $\mathrm{GmbH}$ in Wiesbaden, both of which agreed to join the workshop's Organizing Committee, chaired by J. Müller with U. Dillner providing considerable support.

The workshop aimed to bring together scientists from industry and from research institutions for an informal discussion of the state-of-the-art and developments in order to promote a wider industrial application of thermal microsensors. The current trend towards developing new, cost-effective micromechanical technologies (e.g., CMOS-compatible) for manufacturing thermal microsen- sors was seen as an essential topic. The workshop was therefore of special interest to companies which envisage or foresee the manufacture and application of the various types of thermal microsensors.

There were invited review talks on physical principles (A.W. van Herwaarden, Xensor Integration BV, Delft,), the thermoelectric efficiency of bulk and thin-film materials ( $F$. Völklein, Fachhochschule Wiesbaden), CMOS and micromachining for thermal sensors $(H$. Baltes, ETH, Zurich), and the applications of thermal microsensors (J. Schiferdecker, Heimann Optoelectronics $\mathrm{GmbH}$, Wiesbaden, Germany). A round-table discussion and short contributions presented by 27 of the 52 participants, who came from Belgium, France, Germany, The Netherlands, Russia, Switzerland, the Ukraine, the UK, and the USA, completed the scientific programme. The ample opportunity for discussions and the secluded but attractive venue (a holiday hotel in Oberhof, a health resort in the Thuringian Forest) clearly helped promote a stronger collaboration between fundamental research and industry in the field of thermal microsensors.

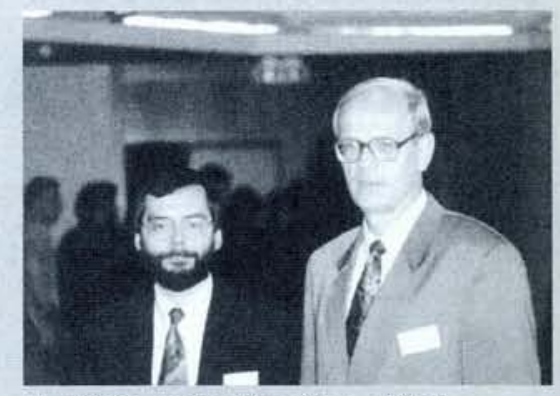

U. Dillner, on the left, with J. Müller

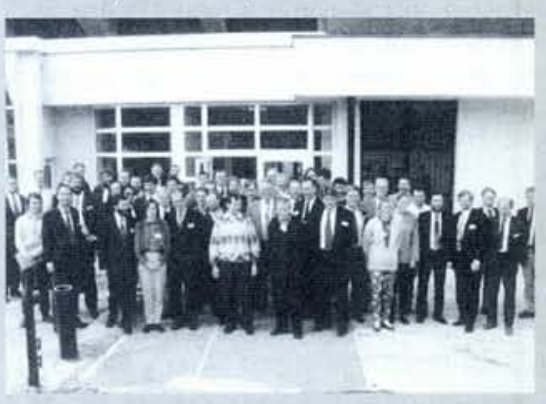

1. Much effort will be spent in evaluating important material properties (thermal conductivity, heat capacity, emissivity, etc.) of the functional layers of thermal microsensors and their dependence on thickness and on quantities which are clearly influenced by the manufacturing technology (e.g., doping concentrations and grain size and other structural parameters). This will lead to more realistic thermal simulations for sensor optimization since the results of such calculations (see cover illustration) depend strongly on the quality of input parameters.

2. The potential of thin-film technologies (e.g., as nanostructured films, quantum wells, superlattices) to improve the thermoelectric efficiency of sensor materials will be studied. 3. The development of thermal microsensor arrays and of multisensing chip configurations will be intensified (an example of the latter is the ETH Zurich group's building control chip shown in Fig. 1d).

4. Micromachined free-standing micro-thermopiles consisting of thin wires and showing very fast response times (less than $20 \mu$ s) will be improved. The aim is to fabricate the sensors as planar arrays.

5. Technological solutions such as SIMOX wafers, CMOS-compatible infra-red absorbers and porous-silicon technology will be introduced into thermal microsensors.

6 . Thermal sensing principles will be employed in designing new chemosensors and biosensors. The arrangement of small drops on thin-film membranes is promising for both microanalytical applications and microcalorimetry as well as for monitoring chemical reactions in small volumes. For instance, recent experiments at the IPHT, Jena, have shown that salt concentrations in solutions can be measured by initiating chemical reaction in droplets supported on a thermopile of the type shown in Fig. 1c.

7. Research dealing with the reliability of thermal microsensors will be intensified since the reliability issue is crucial for the industrial acceptance of new sensor devices.

\section{IPHT, Jena}

\section{Staking Out the Future}

The Institute for Physical High Technology in Jena not only exemplifies the considerable effort spent on reviving applied physics research in east Germany but also provides a fascinating insight into areas where scientific and technical opportunities are believed to lie.
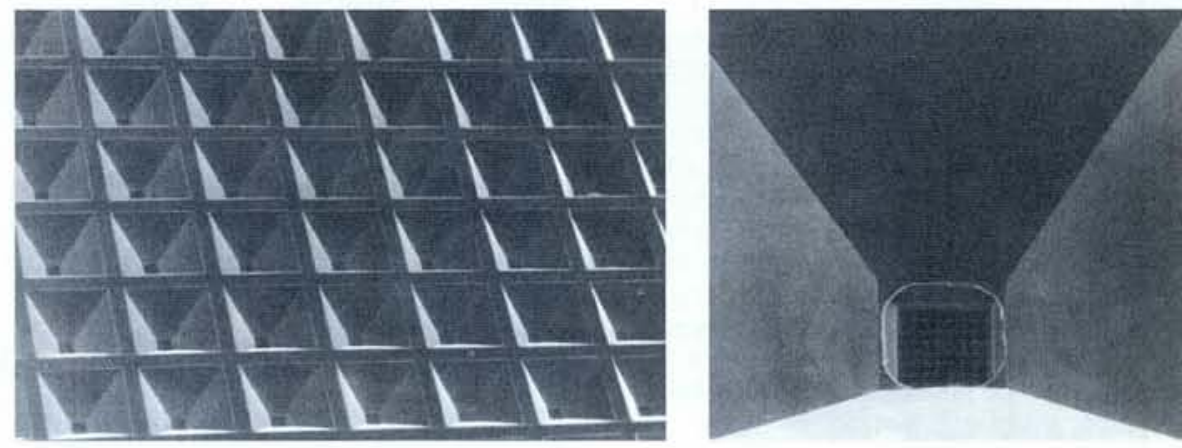

Recent Achievements at the IPHT, Jena. A miniaturised evolution machine for molecular biotechnology: scanning electron micrographs (courtesy: J.M. Köhler) of part of an array, etched in a 4" silicon wafer, of microsieves where each compartment has a $150 \mu \mathrm{m}^{2}$ bottom membrane with 49 pores. A huge variety of different molecules are synthesized in regular way and in parallel by sequentially filling each of the 6000 compartments with a series of chemical reactants. The device can be used for chemical analysis and to determine strategies for synthesizing new molecules. Other IPHT achievements include:

\section{- Optical fibres $\quad 9 \mathrm{~W}$ fibre laser \\ Simulation of polarization-mode dispersion}

- Thin-film thermopiles Precision AC-DC convertors

- Superconductors Cost-effective, $10 \mathrm{GhZ}$ Josephson effect voltage standard

First intrinsic high- $T_{C}$ thin-film Josephson junction

Highest recorded surface resistance for TBCCO-type high- $T_{c}$ films Large melt-textured $\mathrm{YBCO}$ high $-T_{\mathrm{c}}$ samples by levitation melting

The former Physical-Technical Institute (PTI) of the Academy of Sciences of the German Democratic Republic in Jena found itself with a new name, a new legal status, a significantly reduced staff, and a new mission following Germany's reunification. S. Methfessel and B. Elschner steered the institute during the difficult period from 1 January 1992, a few months after the former GDR ceased to exist, to mid-1993. The main challenge was to find a new, stable role that covers the "middle ground" between the industry-oriented Fraunhofer Institute and the essentially basic research orientated MaxPlanck Institute.

The PTI's strengths and weaknesses were evaluated and a plan drawn up which was firmly backed by local and regional governments seeking to re-establish the Jena-Erfurt area as vigorous centre for high-technology industry. Modern precision optics started in Jena and at reunification VEB Carl Zeiss 